\title{
STABILITY AND STRUCTURAL PROPERTIES OF STOCHASTIC STORAGE NETWORKS ${ }^{1}$
}

\author{
by \\ Offer Kella ${ }^{2}$ and Ward Whitt ${ }^{3}$ \\ November 10, 1994 \\ Revision: July 5, 1995 \\ Journal of Applied Probability 33 (1996) 1169-1180 Abstract
}

\begin{abstract}
We establish stability, monotonicity, concavity and subadditivity properties for open stochastic storage networks in which the driving process has stationary increments. A principal example is a stochastic fluid network in which the external inputs are random but all internal flows are deterministic. For the general model, the multi-dimensional content process is tight under the natural stability condition. The multi-dimensional content process is also stochastically increasing when the process starts at the origin, implying convergence to a proper limit under the natural stability condition. In addition, the content process is monotone in its initial conditions. Hence, when any content process with nonzero initial conditions hits the origin, it couples with the content process starting at the origin. However, in general, a tight content process need not hit the origin.
\end{abstract}

Keywords: stability; fluid networks, stationary increments, Lévy process, reflected process, stochastically increasing, tightness, stochastic order, bounds.

\footnotetext{
${ }^{1}$ This work was partially supported by Grant No. 92-00035 from the United-Israel Binational Science Foundation.

${ }^{2}$ Department of Statistics, The Hebrew University of Jerusalem, Mount Scopus, Jerusalem 91905, Israel; email:kella@olive.mscc.huji.ac.il

${ }^{3}$ AT\&T Bell Laboratories, Room 2C-178, 600 Mountain Avenue, Murray Hill, NJ 07974-0636; email: wow@research.att.com
} 


\section{Introduction and Summary}

This work is motivated by an open stochastic fluid network model in which the external inputs are random but all internal flows are deterministic and linear (when the buffers are nonempty). Stochastic fluid networks are natural models when the random fluctuations within the network occur in a shorter time scale than the random fluctuations in the external inputs. Possible applications are to the emerging high-speed communication networks. The deterministic linear internal flows provide sufficient structure that there is hope for obtaining explicit results. Indeed, in Kella and Whitt (1992a) we derived an explicit expression for the steady-state distribution of a two-buffer tandem stochastic fluid network with Lévy external input process (which is never product form).

Further results for more general stochastic fluid networks with Lévy input, all of which are feedforward, are contained in Kella (1993) and Kaspi and Kella (1996). Here we obtain stability and structural results for the general case in which feedback is allowed. We also consider more general input than Lévy processes.

We are primarily interested in the vector content (also known as the workload process or virtual-waiting-time process), which is easily defined in terms of the multi-dimensional reflection map, as in Harrison and Reiman (1981), Chen and Mandelbaum (1989) and Chen and Whitt (1993). Let the $n$-dimensional net input process (driving process) be $X \equiv\{X(t) \mid t \geq 0\}$. Let $P$ be a substochastic $n \times n$ matrix with $P^{n} \rightarrow 0$, so that $(I-P)^{-1}=\sum_{n=0}^{\infty} P^{n}$ is a finite nonnegative matrix, and $P^{\prime}$ is the transpose of $P$. Let $X$ have sample paths that are right continuous with left limits (cádlág). Then the content process $W$ is the $n$-dimensional reflected process

$$
W(t)=\Psi(X)(t)=X(t)+\left(I-P^{\prime}\right) L(t)
$$

where $\Psi$ is the reflection map and $L \equiv\{L(t) \mid t \geq 0\}$ is the minimal nondecreasing nonnegative cádlág process satisfying $W(t) \geq 0$ for all $t \geq 0$. The previous works show that the reflection map taking $X$ into $(L, W)$ is well defined, Lipschitz continuous, and measurable on the function space $D \equiv D\left([0, T], \mathbb{R}^{n}\right)$ of $\mathbb{R}^{n}$-valued cádlág functions with the standard topologies. As a consequence of the direct construction of the content process as the image of the reflection map, limits and rates of convergence for sequences of net input processes (e.g., functional central limit theorems and functional laws of large numbers) translate immediately into corresponding limits and rates for associated sequences of content processes. We will not discuss such limits 
and rates here.

In this paper, we obtain most results in the general setting of (1.1), where $X$ is a general net input process with extra conditions, such as $X$ having stationary increments, but we also obtain some results in the more restrictive setting of a stochastic fluid network, which is the model that motivated this work. The stochastic fluid model has the special net input process defined by

$$
X(t)=X(0)+J(t)-\left(I-P^{\prime}\right) r t,
$$

where $J$ is an $n$-dimensional nondecreasing nonnegative stochastic process, $r$ is a vector of positive constants. In this case we can think of a proportion $P_{i j}$ of all output from buffer $i$ being routed to buffer $j$, while a proportion $1-\sum_{j=1}^{n} P_{i j}$ leaves the network. For the following discussion, consider the fluid model setting of (1.2).

A natural first question is stability. There recently has been great interest and progress on stability results for queueing networks and related models; e.g. see Baccelli and Foss (1994), Dai (1993) and Dai and Weiss (1994), Kumar (1993) and Kumar and Meyn (1994). Here we ask under what conditions do the average inputs over intervals at each buffer converge to appropriate limits? Under what conditions is $\{W(t) \mid t \geq 0\}$ tight, i.e., when is it true that for any $\epsilon>0$ there exists $K_{\epsilon} \in \mathbb{R}_{+}^{n}$ such that $P\left(W(t) \leq K_{\epsilon}\right) \geq 1-\epsilon$ for all $t \geq 0$ ? Moreover, under what conditions is it true that $W(t) \Longrightarrow W$ as $t \rightarrow \infty$, where $W$ is a proper random vector and $\Longrightarrow$ denotes convergence of distribution?

To consider steady-state limits, we will assume that the net input process $X$ has stationary increments with $X(0)=0$, i.e., $X_{s} \equiv\{X(t+s)-X(s) \mid t \geq 0\}$ has a distribution independent of $s$. Under the additional conditions that (i) the $n$ components $J_{i} \equiv\left\{J_{i}(t) \mid t \geq 0\right\}$ of $J \equiv$ $\left(J_{1}, \ldots, J_{n}\right)$ are mutually independent and (ii) $J$ has independent increments, Kaspi and Kella (1996) proved in the feedforward case that $W(t)$ converges to a proper limit, independent of the initial conditions, if

$$
R(E[X(1)-X(0)])=R(E J(1))-r<0
$$

where $R \equiv\left(I-P^{\prime}\right)^{-1}$ or, equivalently, if $\lambda \equiv R(E J(1))<r$.

Condition (1.3) is to be contrasted with the stronger condition

$$
E[X(1)-X(0)]=E J(1)-\left(I-P^{\prime}\right) r<0 .
$$

It is clear that (1.4) implies (1.3), but not conversely; to go from (1.4) to (1.3), we can simply 
premultiply by the matrix $R$, but we cannot go the other way. (Note that $R x$ is strictly positive for each strictly positive $x$.)

In this paper we show for the general case of (1.1) where $X$ has stationary-increments that (1.3) is a sufficient condition for stability, i.e., tightness of the content process for all proper initial distribution. We also establish convergence when the process starts at the origin. In addition, for an appropriate metric, the distance between two processes with two ordered initial conditions is nonincreasing. Nevertheless, convergence with general initial conditions remains a conjecture. Kella (1994) establishes the conjecture for the general initial-conditions case in which the net input process is a Lévy process, and in fact establish convergence in total variation.

Condition (1.3) is the natural stability condition, because $R J(t)$ represents the total required work at each station for all time generated by the input over the interval $[0, t]$. That is, it includes the direct input $J(t)$, the first-routed flow $P^{\prime} J(t)$, the second-routed flow $\left(P^{\prime}\right)^{2} J(t)$ and so forth. Thus $\lambda \equiv R(E J(1))$ in (1.3) should be the vector of input rates of work to each station. We now prove that it is.

It is not difficult to see that (1.3) is essentially necessary and sufficient (except for cases of equality) for $W_{j}(t) / t \rightarrow 0$ as $t \rightarrow \infty$ w.p.1 for all $j$, even in the general setting of (1.1) without any stationary or independence assumptions. In particular, it is easy to establish the following result. We give all proofs in Section 2.

Theorem 1. In the setting of (1.1) with a general net input process $X$, suppose that $X(t) / t \rightarrow$ $\alpha$ as $t \rightarrow \infty$ w.p.1. If $R \alpha \leq 0$, then

$$
W(t) / t \rightarrow 0 \text { as } t \rightarrow \infty \text { w.p. } 1
$$

On the other hand, if $(R \alpha)_{j}>0$ for some $j$, then

$$
\liminf _{t \rightarrow \infty} \sum_{j=1}^{n} W_{j}(t) / t>0 .
$$

We now apply Theorem 1 to deduce that the total (internal plus external) input rates in the fluid network are well defined under the natural generalization of (1.3). Let $I_{j}(t)$ and $D_{j}(t)$ be the total input to buffer $j$ and departure (output) from buffer $j$ in the interval $[0, t]$. Let $I$ and $D$ be the associated vectors. Note that $I$ and $D$ are not well defined in the setting of (1.1) with a general $X$; here we are exploiting the special structure of the fluid model.

Theorem 2. Consider the stochastic fluid model in (1.1) and (1.2). If $J(t) / t \rightarrow \alpha$ as $t \rightarrow \infty$ 
w.p. 1 and $R \alpha<r$, then

$$
\lim _{t \rightarrow \infty} \frac{I(t)}{t}=\lim _{t \rightarrow \infty} \frac{D(t)}{t}=R \alpha \text { as } t \rightarrow \infty \text { w.p.1. }
$$

Condition (1.4) is interesting because it not only is a weaker sufficient condition for the usual notion of stability, but it yields a stronger form of stability. Condition (1.4) states that the net flow rate is negative even when all buffers are simultaneously nonempty. In other words, the flow rate is towards the origin in the interior of the $n$-dimensional positive orthant. For the stochastic fluid networks, under (1.4) we can stochastically bound the content process by considering each component separately with the one-dimensional reflection map. This means that we can conclude that one buffer is stable without directly imposing conditions on the net input to other buffers; i.e., we impose condition (1.4) only for one coordinate $i$. Note that this result depends on the stochastic fluid network structure in (1.2). Also note that we do not need the independent increments condition with (1.4).

Theorem 3. Consider the stochastic fluid setting of (1.2). If $J_{i}$ has stationary increments, if $J_{i}(t) / t \rightarrow E J_{i}(1)$ as $t \rightarrow \infty$ w.p. 1 and if $E J_{i}(1)<\left(\left(I-P^{\prime}\right) r\right)_{i}$, then $\left\{W_{i}(t) \mid t \geq 0\right\}$ is tight.

We can treat the general stationary net input case in (1.1) if all coordinates are simultaneously controlled. For this purpose, we establish an upper bound on a positive linear function of the content process. In fact, we bound the positive linear function of the content process both above and below by content processes whose components evolve as one-dimensional content processes.

Theorem 4. In the general setting of (1.1),

$$
W_{*}(t) \leq R W(t) \leq R W^{*}(t)
$$

where $W_{i}^{*}(t)$ is the one-dimensional content process with net input process $X_{i}(t)$ and $W_{* i}(t)$ is the one-dimensional content process with net input process $(R X(t))_{i}$.

Now if we impose an additional condition, which essentially corresponds to (1.4), then we can establish a stability result. Henceforth we always consider the general setting (1.1) unless we stipulate otherwise.

Theorem 5. If $X$ has stationary increments, if $X_{i}(t) \rightarrow-\infty$ as $t \rightarrow \infty$ w.p.1 for all $i$, and if $X(0)$ is proper, then $\{W(t) \mid t \geq 0\}$ is tight.

To establish convergence, we exploit monotonicity, in the spirit of Loynes (1962). It is easy to see that $W$ is not monotone in $X$, but it turns out that $W$ is monotone in the increments 
of $X$. The following result formalizes this notion.

Theorem 6. Consider $W^{i}=X^{i}+\left(I-P^{\prime}\right) L^{i}, i=1,2,3$. Assume that $X^{2}-X^{1}$ is nonnegative and nondecreasing and that $X^{3}=X^{1}+\left(I-P^{\prime}\right) L^{2}$. Let $e=(1, \ldots, 1)^{\prime}$. Then

(i) $W^{2}(t) \geq W^{1}(t)$ for all $t$,

(ii) $L^{1}-L^{2}$ is nonnegative, nondecreasing and is dominated above by $R\left(X^{2}-X^{1}\right)$,

(iii) $L^{3}=L^{1}-L^{2}$,

(iv) $R\left(W^{2}-W^{1}\right)$ (resp., $\left.e^{\prime} W^{2}-e^{\prime} W^{1}\right)$ is nonnegative and dominated above by $R\left(X^{2}-X^{1}\right)$ (resp., $\left.e^{\prime} X^{2}-e^{\prime} X^{1}\right)$. When $X^{2}-X^{1}$ is a nonnegative constant, then $R\left(W^{2}-W^{1}\right)$ (resp., $\left.e^{\prime} W^{2}-e^{\prime} W^{1}\right)$ is also nonincreasing.

We say that a process $\{Y(t) \mid t \geq 0\}$ is stochastically increasing (SI) if

$$
E f(Y(s)) \leq E f(Y(s+t))
$$

for all positive $s$ and $t$, and for all nondecreasing real-valued functions $f$ for which the expectations are well defined. We now apply Theorem 6 to deduce the following monotonicity result. Theorem 7. If $X$ has stationary increments with $X(0)=0$ (so that $W(0)=0)$, then $\{W(t) \mid t \geq 0\}$ is $S I$.

We can apply Theorems 5 and 7 to establish conditions for convergence under these initial conditions. Let $\Rightarrow$ denote convergence in distribution.

Theorem 8. Under the assumptions of both Theorems 5 and 7 ,

$$
W(t) \Rightarrow W(\infty) \text { as } t \rightarrow \infty
$$

where $W(\infty)$ is a proper random vector.

We now want to say something about other initial conditions. Theorems 6 and 7 allow us to deduce a solidarity result about tightness.

Theorem 9. Let $Y$ have stationary increments and let $X(t)=Y(t)+X(0)$. Then $\{W(t) \mid t \geq$ $0\}$ is tight for all proper distributions of $X(0)$ if and only if it is tight for any one.

We now establish our main tightness result.

Theorem 10. If $X$ has stationary increments with $R(E X(1))<0$ and $X(0)$ is proper, then $\{W(t) \mid t \geq 0\}$ is tight. 
We can combine Theorems 7 and 10 to obtain a condition for convergence.

Corollary. If $X$ has stationary increments with $R(E X(1))<0$ and $X(0)=0$, then $\{W(t) \mid t \geq 0\}$ converges in distribution to a proper limit.

We now deduce additional structural properties of the content process. We can apply Theorem 6 to deduce a concavity result for the mean which extends the one-dimensional results in Kella (1992) and Kella and Sverchkov (1994).

Theorem 11. Under the conditions of Theorem 7, $(R(E W(t)))_{i}$ and $E L_{i}(t)$ are concave functions of $t$ for each $i$. In particular, $e^{\prime} E W(t)$ is a concave function.

We say that $\{Y(t) \mid t \geq 0\}$ is stochastically increasing and subadditive (SIS) if

$$
E f(Y(s+t))<E f(Y(s))+E f(Y(t))
$$

for all positive $s$ and $t$, and for all nondecreasing subadditive real-valued functions $f$ for which the expectations are well defined. (Recall that $f$ is subadditive if $f(x+y) \leq f(x)+f(y)$.) Theorem 12. If $X$ has stationary increments with $X(0)=0$, then $R W(t)$ is SI and SIS.

We do not use the concavity and SIS results further. The SIS result implies that $E f(R W(t)) / t$ converges to a finite limit as $t \rightarrow \infty$ for each subadditive function $f$. For instance, $R(E W(t)) / t$ thus converges to a finite limit as $t \rightarrow \infty$. (The subadditivity could also be used as $t \rightarrow 0$.)

We now consider establishing convergence under general initial conditions. Given Theorems 6 and 7, it suffices to show that $\{W(t) \mid t \geq 0\}$ will hit the origin w.p.1. It will then couple with the process that starts at the origin, which is known to converge (to a possibly infinite limit). However in general $\{W(t) \mid t \geq 0\}$ need not ever visit a neighborhood of the origin.

Example 1. To see that it is possible to have $W(t) \neq(0, \ldots, 0)$ for all $t \geq 0$ under the conditions of Theorem 7, consider a two dimensional case in which either $X_{1}(t+\epsilon)-X_{1}(t)>$ $\delta \epsilon$ or $X_{2}(t+\epsilon)-X_{2}(t)>\delta \epsilon$ for all $t$, where $\epsilon$ and $\delta$ are small positive constants. For example, let

$$
Y_{1}(t)= \begin{cases}\delta, & 3 k \leq t<3 k+2 \\ -1, & 3 k+2 \leq t<3 k+3\end{cases}
$$

and

$$
Y_{2}(t)= \begin{cases}\delta, & 3 k+1 \leq t<3 k+3 \\ -1, & 3 k \leq t<3 k+1\end{cases}
$$

for all nonnegative integers $k$. Let $U$ be uniformly distributed on $[0,3]$. Then $\{Y(t) \mid t \geq$ $0\} \equiv\left\{\left(Y_{1}(t+U), Y_{2}(t+U)\right) \mid t \geq 0\right\}$ is a stationary process on the positive half line, so 
that $X(t) \equiv \int_{0}^{t} Y(u) d u$ is a net input process with stationary increments. It is easy to see that the content process associated with $P^{\prime}=0$ never hits the origin after time 0 ,and yet for $\delta<1 / 2$ it has a proper steady-state distribution. Indeed, eventually $W(t)$ follows the deterministic trajectory shown in Figure 1 with $W(3 k-U)=(2 \delta, 0), W(3 k+1-U)=(0, \delta)$ and $W(3 k+2-U)=(\delta, 2 \delta)$. This steady-state trajectory is reached for

$$
t \geq 3\left(1+\frac{\max \left\{W_{1}(0), W_{2}(0)\right\}}{1-2 \delta}\right) .
$$

By an appropriate choice of units, the limiting trajectory falls outside any neighborhood of the origin.

We can also modify Example 1 to construct two stable content processes which differ only in their initial conditions but do not couple in finite time.

Example 2. We modify Example 1 by letting $P_{12}=P_{21}=\epsilon$ for $0<\epsilon<\delta$. The content process now approaches the deterministic trajectory with $W(3 k-U)=\left(2 \delta-\epsilon+\epsilon^{\prime}, 0\right), W(3 k+1-U)=$ $\left(0, \delta-\epsilon+\epsilon^{\prime}\right)$ and $W(3 k+2-U)=\left(\delta, 2 \delta-\epsilon+\epsilon^{\prime}\right)$, where $\epsilon^{\prime}=\left(2 \delta^{2}-\epsilon \delta\right) /(1+\delta)$. However, unlike Example 1, the content process typically does not reach this cycle in finite time. Suppose one of two content processes starts above another, where they have the same net input process $X$. They move together until they hit a boundary. However, when the lower process is on a boundary and the other is not, the other coordinate of the two processes moves away from each other at rate $\epsilon$. Hence the processes cannot couple on any boundary, although they do get closer in an appropriate metric as they hit the boundaries.

When $X$ is a Lévy process in the setting of (1.2), it is possible to show that $\{W(t) \mid t \geq 0\}$ does hit the origin; see Kella (1994).

\section{Proofs}

Proof of Theorem 1. The assumed convergence $X(t) / t \rightarrow \alpha$ as $t \rightarrow \infty$ w.p.1 is equivalent to the functional strong law of large numbers (FSLLN) $X(n t) / n \rightarrow \alpha t$ as $n \rightarrow \infty$ uniformly on compact sets (u.o.c.) w.p.1.; see Theorem 4 of Glynn and Whitt (1988). By the continuous mapping theorem,

$$
\frac{W(n t)}{n} \rightarrow \Psi(\alpha)(t) \text { u.o.c. as } n \rightarrow \infty \text { w.p.1 }
$$

where $\Psi$ is the multi-dimensional reflection map and $\alpha \equiv\{\alpha t \mid t \geq 0\}$. However, using $L(t)=$ $-R \alpha t$, we see that $\Psi(\alpha)(t)=0$ under the condition $R \alpha<0$. The FSLLN for $W$ in turn implies that $W(t) / t \rightarrow 0$ as $t \rightarrow \infty$ w.p.1. 
Turning to the transient result, note from (1.1) that

$$
R W(t) \geq R X(t), t \geq 0
$$

Hence, if one coordinate of $R X(t)$ grows linearly, so does at least one coordinate of $W(t)$.

Proof of Theorem 2. Note that we have the conservation equation

$$
I_{i}(t)=J_{i}(t)+\sum_{j=1}^{n} D_{j}(t) P_{j i}, \quad t \geq 0 .
$$

Since $I(t)=D(t)+W(t)$

$$
I(t)=\left(I-P^{\prime}\right)^{-1}(J(t)-W(t)) .
$$

Divide by $t$, let $t \rightarrow \infty$ and apply Theorem 1 .

Proof of Theorem 3. Note that $W_{i}$ is bounded above by the case in which all other buffers are assumed to be always nonempty. More formally, note that

$$
\begin{aligned}
W(t) & =\min _{Y}\left\{J(t)-\left(I-P^{\prime}\right) r t+\left(I-P^{\prime}\right) Y(t)\right\} \\
& \leq W^{*}(t) \equiv \min _{Y}\left\{J(t)-\left(I-P^{\prime}\right) r t+Y(t)\right\},
\end{aligned}
$$

where the minimum in each case is over all nondecreasing nonnegative cádlág functions $Y$ satisfying $W(t) \geq 0$. However, since the prefactor $\left(I-P^{\prime}\right)$ of $Y$ is removed in $W^{*}(t)$, we can do the minimization in each coordinate separately, getting $W^{*}(t)=X(t)+L^{*}(t)$, where

$$
L_{i}^{*}(t)=-\inf _{0 \leq s \leq t}\left\{X_{i}(s)^{-}\right\}, t \geq 0,
$$

where $a^{-}=\min \{a, 0\}$. The familiar one-dimensional results, e.g., Theorems 11 and 13, p. 24, of Borovkov (1976), then imply the conclusion.

Proof of Theorem 4. Let $W^{*}(t)=X(t)+L^{*}(t)$ where $L_{i}^{*}(t)$ is defined as in (2.2). Since

$$
W^{*}(t)=X(t)+\left(I-P^{\prime}\right)\left(I-P^{\prime}\right)^{-1} L^{*}(t) \geq 0 \text { for every } t
$$

it follows form the minimality of $L$ that

$$
L(t) \leq\left(I-P^{\prime}\right)^{-1} L^{*}(t), t \geq 0
$$

Therefore,

$$
\tilde{W}(t) \equiv R W(t) \leq R W^{*}(t), t \geq 0 .
$$

Similarly, let $\tilde{W}_{*}=\tilde{X}+\tilde{L}_{*}$, where $\tilde{X}=R X$ and $\tilde{L}_{* i}=-\inf _{0 \leq s \leq t} \tilde{X}_{i}(s)$, and conclude that $\tilde{L}_{*} \leq \tilde{L}$. Hence, we have the lower bound as well. 
Proof of Theorem 5. Under the extra condition, we can apply the familiar one-dimensional result, see Theorems 11 and 13, p. 24, of Borovkov (1976), to deduce that $W_{i}^{*}(t) \Rightarrow W_{i}^{*}(\infty)$ as $t \rightarrow \infty$ for each $i$. Hence, $\left\{(R W(t))_{i} \mid t \geq 0\right\}$ is tight for each $i$, which in turn implies that the vector-valued process $\{R W(t) \mid t \geq 0\}$ is tight. This means that every sequence $\left\{R W\left(t_{k}\right): k \geq 1\right\}$ has a convergent subsequence to a proper limit. By the continuous mapping theorem with the map $I-P^{\prime}$, the same is true for $\{W(t) \mid t \geq 0\}$. Hence, $\{W(t) \mid t \geq 0\}$ itself is tight.

Proof of Theorem 6. (i) It suffices to consider a single step in a discrete-time approximation. This is so because the sample path of a cádlág net input process $X$ can be represented as the limit of discrete-time net input processes. The $n^{\text {th }}$ approximation can be $X^{n}(t)=X(\lfloor n t\rfloor / n), t \geq 0$, where $\lfloor x\rfloor$ is the integer part of $x$, which is constant except for jumps at times $k / n$. If $X$ is not continuous, then $X^{n}$ need not converge to $X$ in the topology of uniform convergence on compact intervals, but $X^{n}$ will converge to $X$ in the usual topologies for cádlág functions, see Chapter 3 of Billingsley (1968). The same will be true for the content processes because the reflection map is continuous in these other topologies, see Sections 2 of Chen and Whitt (1993). Convergence in these topologies for cádlág functions implies pointwise convergence at all continuity points, which in turn implies preservation of the inequalities. Since $X^{2}-X^{1}$ is nonnegative and nondecreasing, so is $X^{2 n}-X^{1 n}$.

For the discrete-time processes, we proceed by induction. Thus, it suffices to consider a single step in a discrete-time process. However, even a single step is somewhat complicated to analyze. For any $w \in \mathbb{R}^{n}$, the single-step reflection map is defined by

$$
\psi(w)=w+\left(I-P^{\prime}\right) l
$$

where $l$ is the minimum nonnegative vector such that $\psi(w)$ is nonnegative. We now show how to represent $\psi$ as the limit of a sequence of operators. Let

$$
T(w)=w^{+}+P^{\prime} w^{-}
$$

and let $T^{n}$ be the $n$-fold iteration of the operator $T$.

Lemma 1. The one-step reflection operator $\Psi$ in (2.7) satisfies

$$
\psi(w)=\lim _{n \rightarrow \infty} T^{n}(w)
$$

with

$$
l=-\sum_{k=0}^{\infty}\left(T^{k}(w)\right)^{-}=\left(P^{\prime} l-w\right)^{+}
$$


Proof of Lemma 1. Let $w_{n}=T^{n}(w)$ with $w_{0}=w$. Clearly $w_{n}-w_{n-1}=-\left(I-P^{\prime}\right) w_{n-1}^{-}$, so that

$$
w_{n}=w_{0}-\left(I-P^{\prime}\right) \sum_{k=0}^{n-1} w_{k}^{-} .
$$

Since $w_{n}=w_{n-1}^{+}+P^{\prime} w_{n-1}^{-}, P^{\prime} w_{n-1}^{-} \leq w_{n} \leq w_{n-1}^{+}$, which in turn implies that $P^{\prime} w_{n-1}^{-} \leq$ $w_{n-1}^{-} \leq 0$ and $w_{n}^{+} \leq w_{n-1}^{+}$. Hence, $0 \geq w_{n}^{-} \geq\left(P^{\prime}\right)^{n} w_{0}^{-} \rightarrow 0$ as $n \rightarrow \infty$, so that $w_{n} \rightarrow w_{\infty} \geq 0$.

To show that $l=\left(P^{\prime} l-w\right)^{+}$, recall that $-P^{\prime} w_{k}^{-}=w_{k}^{+}-w_{k+1}$ and write

$$
\begin{aligned}
\left(P^{\prime} l-w\right)^{+} & =\lim _{n \rightarrow \infty}\left(P^{\prime} \sum_{k=0}^{n-1}\left(-w_{k}^{-}\right)-w_{0}\right)^{+} \\
& =\lim _{n \rightarrow \infty}\left(\sum_{k=0}^{n-1} w_{k}^{+}-\sum_{k=1}^{n} w_{k}-w_{0}\right)^{+} \\
& =\lim _{n \rightarrow \infty}\left(-w_{n}^{+}-\sum_{k=0}^{n} w_{k}^{-}\right)^{+} \\
& =\left(-w^{+}+l\right)^{+}=(-w+l)^{+}
\end{aligned}
$$

For $i$ such that $w_{i}=0,(-w+l)_{i}^{+}=l_{i}$. For $i$ such that $w_{i}>0$, we have from $w_{n}^{+} \leq w_{n-1}^{+}$that $w_{n i}^{+}>0$ for all $i$, which implies that $l_{i}=0$. Then $(-w+l)_{i}^{+}=0$. We thus see that $l$ in $(2.10)$ is the $l$ in $(2.7)$.

We now return to the proof of Theorem 6 . From (2.8), it is elementary that the operator $T$ is monotone in $w$. By (2.9), we see that $\psi$ is also monotone in $w$.

(ii) We start by establishing some properties of the regulator process $L$. For a cádlág process $Y$, denote $U^{t}(Y)=\left(U_{i}^{t}(Y)\right)$, where $U_{i}^{t}(Y)=\sup _{0 \leq s \leq t} Y_{i}(s)^{+}$with $a^{+}=\max (a, 0)$. We note that $U^{t}(Y)$ is nondecreasing in both $Y$ and $t$ and that for every constant vector $c, U^{t}(Y+c) \leq U^{t}(Y)+c^{+}$where $c^{+}=\left(c_{i}^{+}\right)$. For a given cádlág $K$, let $T^{Y} K(t)=U^{t}\left(P^{\prime} K-Y\right)$. Then it is well known and easy to check that $\left(T^{Y}\right)^{N}$ is a contraction for some large enough $N$ (on the space of cádlág functions with the uniform topology). Hence there is a unique $L^{Y}$ such that $L^{Y}=T^{Y} L^{Y}$ for every cádlág $K,\left(T^{Y}\right)^{n} K \rightarrow L^{Y}$ as $n \rightarrow \infty$. In fact, when $Y(0) \geq 0, L^{Y}$ is also the minimal nondecreasing process satisfying $W^{Y}(t) \equiv Y(t)+\left(I-P^{\prime}\right) L^{Y}(t) \geq 0$ for which $L^{Y}(0)=0$. It also satisfies $\int_{0}^{\infty} W_{i}^{Y}(t) d L_{i}^{Y}(t)=0$ for all $i$. For an excellent in depth study of this material and for further references, see Chen and Mandelbaum (1989). The following extends (2.9) of Chen and Whitt (1993).

Lemma 2. Let $X$ and $Y$ be cádlág. Then, for every $t \geq 0$,

$$
-R U^{t}(X-Y) \leq L^{X}(t)-L^{Y}(t) \leq R U^{t}(Y-X) .
$$

In particular, if $Y(t)=w+X(t)$ for some $w \geq 0$, then

$$
L^{Y}(t) \leq L^{X}(t) \leq L^{Y}(t)+R w .
$$


Proof. Set $L^{Y, n} \equiv\left(T^{Y}\right)^{n} L^{X}$ and note that $L^{Y, n} \rightarrow L^{Y}$ as $n \rightarrow \infty$. $\left(\left(T^{Y}\right)^{0}\right.$ being the identity). Then, assuming that $L^{X} \leq L^{Y, n}(t)+\sum_{i=0}^{n-1}\left(P^{\prime}\right)^{i} U^{t}(Y-X)$,

$$
\begin{aligned}
L^{X}(t)=T^{X} L^{X}(t) & =U^{t}\left(P^{\prime} L^{X}-X\right) \\
& \leq U^{t}\left(P^{\prime} L^{Y, n}-Y+P^{\prime} \sum_{i=0}^{n-1}\left(P^{\prime}\right)^{i} U^{t}(Y-X)+(Y-X)\right) \\
& \leq U^{t}\left(P^{\prime} L^{Y, n}-Y+\sum_{i=0}^{n}\left(P^{\prime}\right)^{i} U^{t}(Y-X)\right) \\
& \leq U^{t}\left(P^{\prime} L^{Y, n}-Y\right)+\sum_{i=0}^{n}\left(P^{\prime}\right)^{i} U^{t}(Y-X) \\
& =L^{Y, n+1}(t)+\sum_{i=0}^{n}\left(P^{\prime}\right)^{i} U^{t}(Y-X),
\end{aligned}
$$

where the last equality follows from $L^{Y, n+1}(t)=T^{Y} L^{Y, n}(t)=U^{t}\left(P^{\prime} L^{Y, n}-Y\right)$. Letting $n \rightarrow$ $\infty$ establishes the right inequality in (2.11). The left inequality follows by symmetry. The inequalities in (2.12) follow from (2.11).

Now, returning to the proof of (ii), $0 \leq L^{1}-L^{2} \leq R\left(X^{2}-X^{1}\right)$ follows from (2.11). To show that $L^{1}-L^{2}$ is nondecreasing, it suffices to argue that for every nonnegative $s, t$ we have that $L^{1}(t+s)-L^{1}(s) \geq L^{2}(t+s)-L^{2}(s)$. Note that $L^{i}(t+s)-L^{i}(s)$ is the regulator process when the net input process is $\left\{W^{i}(s)+X^{i}(s+t)-X^{i}(s) \mid t \geq 0\right\}$. By assumption, $X^{2}(s+t)-X^{2}(s) \geq X^{1}(s+t)-X^{1}(s)$ for every $t$ and, from (i), $W^{2}(s) \geq W^{1}(s)$. Therefore, the result once again follows from the left side of (2.11) (which is zero in this case).

(iii) We note that

$$
\begin{aligned}
& 0 \leq W^{3}=X^{1}+\left(I-P^{\prime}\right)\left(L^{2}+L^{3}\right) \\
& 0 \leq W^{1}=X^{3}+\left(I-P^{\prime}\right)\left(L^{1}-L^{2}\right) .
\end{aligned}
$$

The fact that $L^{2}+L^{3} \geq L^{1}$ follows by minimality and holds for any choice of $X^{1}$ and $X^{2}$. From (ii), $L^{1}-L^{2}$ is nonnegative and nondecreasing, hence $L^{1}-L^{2} \geq L^{3}$ by minimality as well, and the result follows.

(iv) This final result follows from $R W^{i}=R X^{i}+L^{i}$, (ii) and the fact that $e^{\prime}\left(I-P^{\prime}\right) \geq 0$, as $P$ is substochastic.

Proof of Theorem 7. Note that $W(s+t)$ is equal to $W^{s}(t)$, which we define as the content at time $t$ with initial content $W(s)$ and net input process $X_{s}(t)=X(t+s)-X(s)$. Since $X$ has stationary increments, $X_{s}$ is distributed the same as $X$. Also $W(t)$ has the same law as the content $W_{s}(t)$ which we define as the content with initial condition 0 and net input process $X_{s}$. By Theorem $6, W_{s}(t) \leq W^{s}(t)$ for all $t$. Hence, $W(t)$ is stochastically smaller than $W(t+s)$. 
Proof of Theorem 9. Note that $\{W(t) \mid t \geq 0\}$ is tight if and only if $\{R W(t) \mid t \geq 0\}$ is tight. Use Theorem 6 to show that the processes $R W(t)$ starting at $X(0)$ and 0 differ by at most $R X(0)$. Hence they either are both tight or both nontight.

Proof of Theorem 10. Let $X$ have stationary increments with $R x<0$, where $x=E X(1)$. Let $y>x$ be such that $R y<0$. Set $X^{\prime}(t)=X(t)-y t$, then $X^{\prime}$ has stationary increments with $E X^{\prime}(1)=x-y<0$. Let

$$
\begin{aligned}
W(t) & =W(0)+X(t)+\left(I-P^{\prime}\right) L(t) \\
W^{\prime}(t) & =W(0)+X^{\prime}(t)+L^{\prime}(t) \\
W^{\prime \prime}(t) & =y t+\left(I-P^{\prime}\right) L^{\prime \prime}(t),
\end{aligned}
$$

where $(W, L),\left(W^{\prime}, L^{\prime}\right)$ and $\left(W^{\prime \prime}, L^{\prime \prime}\right)$ satisfy the dynamic complementarity conditions, as in (1.2). Since $\left(I-P^{\prime}\right)^{-1} y<0, W^{\prime \prime}(t)=0$ for all $t \geq 0$. From

$$
0 \leq W^{\prime}(t)=W^{\prime}(t)+W^{\prime \prime}(t)=W(0)+X(t)+L^{\prime}(t)+\left(I-P^{\prime}\right) L^{\prime \prime}(t)
$$

we have by minimality that $\left(I-P^{\prime}\right)^{-1} L^{\prime}(t)+L^{\prime \prime}(t) \geq L(t)$. Therefore, $\left(I-P^{\prime}\right)^{-1} W(t) \leq$ $\left(I-P^{\prime}\right)^{-1} W^{\prime}(t)$ for every $t$. By Theorem $5, W^{\prime}$ is tight. Hence, so is $W$.

Proof of Theorem 11. We want to show that

$$
R(E W(t+s))-R(E W(s))
$$

is nonincreasing in $s$ for all $t$. This follows from Theorem 9, because $W(s+t)=W^{s}(t)$, the content at $t$ with initial condition $W(s)$ and net input process $X_{s}(t)=X(s+t)-X(s)$, while $W_{s}(t)$ is distributed the same as the content process with initial condition 0 and net input process $X_{s}$ (because the distribution of $X_{s}$ is independent of $s$ ).

Proof of Theorem 12. The SI property follows immediately from Theorem 7 , but it can also be easily deduced directly. Let $\tilde{W}_{s}(t)=R W_{s}(t)$, where $W_{s}(t)$ is the content at $t$ with initial condition $W(s)$ and net input process $X_{s}(t)=X(s+t)-X(s)$. Then, from Theorem 6 (iv), we see that

$$
\tilde{W}_{s}(t) \leq \tilde{W}_{0}(s+t) \leq \tilde{W}_{0}(s)+\tilde{W}_{s}(t)
$$

so that for nondecreasing functions $\operatorname{Ef}\left(\tilde{W}_{0}(t)\right)=\operatorname{Ef}\left(\tilde{W}_{s}(t)\right) \leq E f\left(\tilde{W}_{0}(s+t)\right)$ and for nondecreasing subadditive functions

$$
\begin{aligned}
\operatorname{Ef}\left(\tilde{W}_{0}(s+t)\right) \leq E f\left(\tilde{W}_{0}(s)+\tilde{W}_{s}(t)\right) & \leq E f\left(\tilde{W}_{0}(s)\right)+\operatorname{Ef}\left(\tilde{W}_{s}(t)\right) \\
& =E f\left(\tilde{W}_{0}(s)\right)+\operatorname{Ef}\left(\tilde{W}_{0}(t)\right),
\end{aligned}
$$


and the proof is complete. 


\section{References}

Baccelli, F. and Foss, S. 1994. Ergodicity of Jackson-type queueing networks. Queueing Systems, 17 5-72.

Billingsley, P. 1968. Convergence of Probability Measures, Wiley, New York.

Borovkov, A. A. 1976. Stochastic Processes in Queueing Theory, Springer-Verlag, New York.

Chen, H. and Mandelbaum, A. 1989. Leontief systems, RBV's and RBM's, Proceeding of the Imperial College Workshop on Applied Stochastic Processes, Davis, M. H. A. and Elliotte, R. J. (eds.), Gordon and Breach Science publishers.

Chen, H. and Whitt, W. 1993. Diffusion approximations for open queueing networks with service interruptions. Queueing Systems 13 335-359.

Dai, J. 1993. On the positive Harris recurrence for multiclass queueing networks: a unified approach via fluid limits. preprint.

Dai, J. and Weiss, G. 1994. Stability and instability of fluid models for certain re-entrant lines. preprint.

Glynn, P. W. and Whitt, W. 1988. Ordinary CLT and WLLN versions of $L=\lambda W$. Math. Oper. Res. 13 674-692.

Harrison, J. M. and Reiman, M. I. 1981. Reflected Brownian motion on an orthant. Ann. Prob. 9 302-308.

Kaspi, H. and Kella, O. 1996. Stability of feed-forward fluid networks with Lévy input. J. Appl. Prob. 33, to appear.

Kella, O. 1992. Concavity and reflected Lévy processes. J. Appl. Prob. 29 209-215.

Kella, O. 1993. Parallel and tandem fluid networks with dependent Lévy inputs. Ann. Appl. Prob. 3 682-695.

Kella, O. 1994. Stability and non-product form of stochastic fluid networks with Lévy inputs. preprint. 
Kella, O. and Sverchkov, M. 1994. On concavity of the mean function and stochastic ordering for reflected processes with stationary increments. J. Appl. Prob. 31 1140-1142.

Kella, O. and Whitt, W. 1992a. A tandem fluid network with Lévy input. In Queues and Related Models, I. Basawa and U. Bhat (eds.), Oxford Press, 112-128.

Kella, O. and Whitt, W. 1992b. Useful martingales for stochastic storage processes with Lévy input. J. Appl. Prob. 29 296-403.

Kumar, P. R. 1993. Re-entrant lines. Queueing Systems 13 87-110.

Kumar, P. R. and Meyn. S. P. 1994. Stability of queueing networks and scheduling policies. preprint.

Loynes, R. M. 1962. The stability of a queue with non-dependent inter-arrival and service times. Proc. Camb. Phil. Soc. 58 497-520. 
Figure 1. The limiting cycle for Example 1. 